\title{
The Initial Value Problem for the Equations of Magnetohydrodynamic Type in Non-Cylindrical Domains
}

\author{
M.A. ROJAS-MEDAR and R. BELTRAN-BARRIOS
}

\begin{abstract}
By using the spectral Galerkin method, we prove the existence of weak solutions for a system of equations of magnetohydrodynamic type in non-cylindrical domains.
\end{abstract}

\section{INTRODUCTION}

In several situations the motion of incompressible electrical conducting fluids can be modelled by the so called equations of magnetohydrodynamics, which correspond to the Navier-Stokes' equations coupled with the Maxwell's equations. In the case where there is free motion of

1991 Mathematics Subject Classification: 35Q10, 76D99, 35D05

Servicio publicaciones Univ. Complutenge. Madrid, 1995. 
heavy ions, not directly due to the electric field (see Schlüter [14] and Pikelner [12]), these equations can be reduced to the following form:

$$
\left\{\begin{array}{l}
\frac{\partial u}{\partial t}-\frac{\eta}{\rho_{m}} \Delta u+u . \nabla u-\frac{\mu}{\rho_{m}} h . \nabla h=f-\frac{1}{\rho_{m}} \nabla\left(p^{*}+\frac{\mu}{2} h^{2}\right), \\
\frac{\partial h}{\partial t}-\frac{1}{\mu \sigma} \Delta h+u . \nabla h-h \cdot \nabla u=-\operatorname{grad} \omega \\
\operatorname{div} u=0 \\
\operatorname{div} h=0
\end{array}\right.
$$

together with suitable boundary and initial conditions.

Here, $u$ and $h$ are respectively the unknown velocity and magnetic fields; $p^{*}$ is the unknown hydrostatic pressure; $\omega$ is an unknown function related to the motion of heavy ions (in such way that the density of electric current, $j_{0}$, generated by this motion satisfies the relation rot $\left.j_{0}=-\sigma \nabla \omega\right) ; \rho_{m}$ is the density of mass of the fluid (assumed to be a positive constant); $\mu>0$ is the constant magnetic permeability of the medium; $\sigma>0$ is the constant electric conductivity; $\eta>0$ is the constant viscosity of the fluid; $f$ is an given external force field.

In this paper we will consider the problem of existence of weak solutions for the problem (1.1) in a time-dependent domain of $\mathbb{R}^{\mathbf{n}} \times$ $(0, T), n \geq 2,0<T<+\infty$.

To (1.1) we append the following initial and boundary conditions

$$
\begin{gathered}
\left.u\right|_{\partial Q}=0 \text { and }\left.\quad h\right|_{\partial Q}=0 \text { for all } t \\
u(0)=u_{0} \quad \text { and } \quad h(0)=h_{0} \quad \text { in } Q(0),
\end{gathered}
$$

where $u_{0}$ and $h_{0}$ are given functions. In (1.1), the diferential operator $\nabla$ and $\Delta$ are the usual gradient and Laplace operator, respectively.

The main goal in this paper is to show existence of weak solutions for the initial value problem (1.1)-(1.3). Our strategy for setting this question consists of transforming problem (1.1)-(1.3) into another initial boundary-value problem in a cylindrical domain whose sections are not time-dependent. This is done by means of a suitable change of variable. 
Next, this new initial value problem is treated using Galerkin approximation. We conclude returning to $Q$ using the inverse of the above change of variable. This technicality was introduced by Dal Passo and Ughi [4] to study a certain class of parabolic equations in non-cylindrical domains.

We feel that it is appropriate to cite some earlier works on the initial value problem (1.1)-(1.3) and to locate our contribution therein. The cylindrical case of (1.1)-(1.3) has been studied by some authors. Among them, let us mention the paper of Lassner [7], Boldrini and Rojas-Medar [2], Rojas-Medar and Boldrini [13].

Lassner [7] by making the use of semigroup techniques as the ones in Fujita and Kato [5] to show the local existence and uniqueness of strong solution. The more constructive spectral Galerkin method was used by Boldrini and Rojas-Medar [2], [13] to obtain local, global existence and uniqueness of strong solutions. The above authors working in $\mathbb{R}^{\mathbf{n}}$ with $n=2$ or 3 .

A mathematical study of the problem (1.1)-(1.3) in a non-cylindrical domain was not done, however, it has to be pointed out that similar timedependent problem but for the Navier-Stokes and Boussinesq problems have been studied by many different authors. This is the case, for instance of the works Lions [9], [8], Fujita and Sauer [6], Oeda [10], Otani and Yamada [11], Conca and Rojas-Medar [3]. In particular, we would like to emphasize that the arguments of the mentioned authors demand that $Q(t)$ be nondecreasing with respect to $t$ (see Lions [9], problem 11.9, p. 426); except the work of Conca and Rojas-Medar [3]. (1.3).

In this work, we will adapt the technique by [3] to the system (1.1)-

The paper is organized as follows. In Section 2 we introduce various functional spaces and state the theorems. Section 3 and 4 deal with their proofs.

\section{FUNCTION SPACES AND PRELIMINAIRES}

The functions in the paper are either $\mathbb{R}$ or $\mathbb{R}^{\mathbf{n}}$-valued and we will not distinguish these two situations in our notations. To which case we refer will be clear from the context. 
Let $\Omega$ be a bounded domain in $\mathbb{R}^{\mathbf{n}}(\mathrm{n} \geq 2$ ) with smooth boundary $\partial \Omega$. We write simply $\|u\|$ for $L^{2}(\Omega)$-norm. The inner product in $L^{2}(\Omega)$ is denoted by $(u, v)$. The solenoidal function space is defined as usual

$$
\begin{aligned}
C_{0, \sigma}^{\infty}(\Omega) & =\left\{\varphi \in C_{0}^{\infty}(\Omega) \mid \operatorname{div} \varphi=0\right\} \\
H(\Omega) & =\text { the completion of } C_{0, \sigma}^{\infty}(\Omega) \text { under the } L^{2}(\Omega)-\text { norm, } \\
V_{s}(\Omega) & =\text { the completion of } C_{0, \sigma}^{\infty}(\Omega) \text { under the } H^{s}(\Omega)-\text { norm }
\end{aligned}
$$

where $H^{s}(\Omega)$ denote the usual Sobolev'space with $s \in \mathbb{R}$.

The norm and inner product in $H(\Omega)$ and $V_{s}(\Omega)$ are:

$$
(f, g)=\sum_{i=1}^{n} \int_{\Omega} f_{i} g_{i} d x, \quad\|f\|=(f, f)^{1 / 2}
$$

and

$$
(u, v)_{s}=\sum_{i=1}^{n}\left(u_{i}, v_{i}\right)_{H^{*}}, \quad\|u\|_{s}=(u, u)_{s}^{1 / 2}
$$

where $\left(u_{i}, v_{i}\right)_{H^{s}}$ is the standard inner product of $H^{s}(\Omega) ;\left(V_{s}(\Omega)\right)^{\prime}$ denotes the topological dual of $V_{s}(\Omega)$.

In particular, we denote

$$
V_{1}(\Omega)=V(\Omega) \text { and } \quad\|u\|_{1}=\|\nabla u\|
$$

We will use other standard notations and terminology; for them, we refer to Adams[1] and Temam [15].

Let $r$ be a real-valued function which is of $C^{1}$-class on the interval $[0, T]$ such that,

$$
r\left(t_{0}\right)=\min \{r(t) \mid 0 \leq t \leq T\}>0
$$

(this condition is essential). 
The time-dependent space domain $Q(t)$ is a bounded set in $\mathbb{R}^{n}$ defined by

$$
Q(t)=\left\{x \in \mathbb{R}^{\mathrm{n}} /|x|<r(t), 0 \leq t \leq T\right\}
$$

where $|+|$ denotes the usual norm in $\mathbb{R}^{\mathrm{n}}$. Its boundary is

$$
\partial Q(t)=\left\{x \in \mathbb{R}^{\mathbf{n}}|| x \mid=r(t), 0 \leq t \leq T\right\} .
$$

It is worth noting that such domains $Q(t) 0 \leq t \leq T$, generate a non-cylindrical time-dependent domain of $\mathbb{R}^{\mathrm{n}} \times \mathbb{R}$ :

$$
Q=\bigcup_{0<t<T} Q(t) \times\{t\}
$$

In such conditions, we can now define a notion of weak solution for (11)-(13):

Definition 2.1. We shall say that a couple of functions $(u, h)$ defined in $Q$ is a weak solution of (1.1)-(1.3) iff

(i) $u, h \in L^{2}(0, T ; V(Q(t))) \cap L^{\infty}(0, T ; H(Q(t)))$

(ii) $-\int_{Q}\left[\alpha u \cdot \varphi_{t}-\nu \sum_{i=1}^{n} \nabla u_{i} \nabla \varphi_{i}+\alpha \sum_{i j=1}^{n} u_{j} \frac{\partial \varphi_{i}}{\partial x_{j}} u_{i}-\sum_{i j=1}^{n} h_{j} \frac{\partial \varphi_{i}}{\partial x_{j}} h_{i}\right] d x d t=$ $\alpha \int_{Q} f \varphi d x d t$

(iii) $-\int_{Q}\left[h \cdot \tilde{\varphi}_{t}-\gamma \sum_{i=1}^{n} \nabla h_{i} \nabla \tilde{\varphi}_{i}+\sum_{i_{j}=1}^{n} u_{j} \frac{\partial \bar{\varphi}_{i}}{\partial x_{j}} h_{i}-\sum_{i j=1}^{n} h_{j} \frac{\partial \tilde{\varphi}_{i}}{\partial x_{j}} u_{i}\right] d x d t=0$ for all $\varphi, \bar{\varphi} \in C_{0}^{1}(Q)$ with $\operatorname{div} \varphi=\operatorname{div} \bar{\varphi}=0$, the suffix $t$ denotes the operator $\frac{\partial}{\partial t}$ (derivatives with respect to $t$ will sometimes also be denoted by $a^{\prime}$ or simply by $\left.d / d t\right)$.

(iv) $u(0)=u_{0}, \quad h(0)=h_{0}$.

where put $\alpha=\frac{p m}{\mu} \nu=\frac{\eta}{\mu}$ and $\gamma=\frac{1}{\mu \sigma}$.

Remark 2.2. In this definition the initial conditions (iv) have the usual meaning; see for example, Lions [8].

The main result of our article is 
Theorem 2.3. If $f \in L^{2}(Q), u_{0}, h_{0} \in H(Q(0))$, then there exists a weak solution of problem (1.1)-(1.3) for any time interval $[0, T]$.

Theorem 2.4. If $n=2$, the solution $(u, h)$ obtained in the Theorem 2.3 is unique. Moreover $u$ and $h$ are almost everywhere equal to functions continuous from $[0, T]$ into $H$ and

$$
\begin{gathered}
u(t) \rightarrow u_{0} \quad \text { in } H, \quad \text { as } t \rightarrow 0 \\
h(t) \rightarrow h_{0} \quad \text { in } H, \quad \text { as } t \rightarrow 0 .
\end{gathered}
$$

\section{PROOF OF THEOREM 2.3.}

Let us introduce the transformation $\tau: Q \rightarrow U$, given by

$$
\tau(x, t)=\left(\frac{x}{r(t)}, t\right)
$$

where $U \equiv D \times(0, T)$ and $D=\left\{x \in \mathbb{R}^{\mathbf{n}}|| x \mid \leq 1\right\}$. Since $r(t)$ is a $C^{\mathbf{1}}$. function which verifies (2.1), we see easily that $\tau$ is a diffeomorphism and that its inverse $\tau^{-1}: U \rightarrow Q$ satisfies

$$
\tau^{-1}(y, t)=(r(t) y, t) .
$$

We also define

$$
\begin{aligned}
v(y, t) & =u(y r(t), t), \\
b(y, t) & =h(y r(t), t), \\
q(y, t) & =p(y r(t), t), \\
\xi(y, t) & =\omega(y r(t), t), \\
J(y, t) & =f(y r(t), t), \\
v_{0}(y) & =u_{0}(y r(0)), \\
b_{0}(y) & =h_{0}(y r(0)) .
\end{aligned}
$$


By using (3.2), the system on $Q$ (1.1)-(1.3) is transformed into the system:

$$
\begin{gathered}
\alpha v^{\prime}-\frac{\nu}{r(t)} \Delta v+\frac{\alpha}{r(t)} \sum_{i=1}^{h} v_{i} \frac{\partial v}{\partial y_{i}}-\frac{1}{r(t)} \sum_{i=1}^{n} b_{i} \frac{\partial b}{\partial y_{i}}= \\
\alpha J+\frac{\mu}{r(t)} \nabla q+\alpha \frac{r^{\prime}(t)}{r(t)} \sum_{i=1}^{n} \frac{\partial v}{\partial y_{i}} y_{i} \\
b^{\prime}-\frac{\gamma}{r(t)} \Delta b+\frac{1}{r(t)} \sum_{i=1}^{n} v_{i} \frac{\partial b}{\partial y_{i}}-\frac{1}{r(t)} \sum_{i=1}^{n} b_{i} \frac{\partial v}{\partial y_{i}}= \\
\frac{r^{\prime}(t)}{r(t)} \sum_{i=1}^{n} \frac{\partial b}{\partial y_{i}} y_{i}+\frac{1}{r(t)} \nabla \xi \\
v(0, y)=v_{0}(y) \\
b(0, y)=b_{0}(y)
\end{gathered}
$$

on the cylindrical domain $U=D \times(0, T)$.

On the other hand, let us set

$$
\begin{aligned}
c(v, w) & =\sum_{i, j=1}^{n} \int_{D} \frac{\partial v_{i}}{\partial y_{j}} y_{j} w_{i} d y \\
a(v, w) & =\sum_{i=1}^{n} \int_{D} \frac{\partial v_{j}}{\partial y_{i}} \frac{\partial w_{i}}{\partial y_{i}} \\
B(u, v, w) & =\sum_{i, j=1}^{n} \int_{D} u_{j} \frac{\partial v_{i}}{\partial y_{j}} w_{i} d y
\end{aligned}
$$

for vector-valued functions $u, v$ and $w$ for which the integrals are welldefined. 
The notation of weak solution for (3.3)-(3.6) is completely similar to the ones for (1.1)-(1.3).

To prove the existence of solutions of the transformed system (3.3)(3.6) we will use the spectral Galerkin method. That is; we fix $s=\frac{n}{2}$ and we consider the Hilbert special basis $\left\{w^{i}(x)\right\}_{i=1}^{\infty}$ of $V_{s}(D)$, whose elements we will choose as the solution of the following spectral problem

$$
\left(w^{i}, v\right)_{s}=\lambda_{i}\left(w^{i}, v\right) \quad \forall v \in V_{s}(D)
$$

Let $V^{k}$ be the subspace of $V_{s}(D)$ spanned by $\left\{w^{1}, \ldots, w^{k}\right\}$. For every $k \geq 1$, we define approximations $v^{k}, b^{k}$ of $v$ and $b$, respectively, by means of the following finite expansions:

$$
v^{k}=\sum_{i=1}^{k} c_{i k}(t) w^{i}(x) \in V^{k} \quad t \in(0, T)
$$

and

$$
b^{k}=\sum_{i=1}^{k} d_{i k}(t) w^{i}(x) \in V^{k} \quad t \in(0, T)
$$

where the coefficients $\left(c_{i k}\right)$ and $\left(d_{i k}\right)$ will be calculated in such a way that $v^{k}$ and $b^{k}$ solve the following approximations of system (3.3)-(3.6):

$$
\begin{aligned}
& \alpha\left(v_{t}^{k}, \phi\right)+\frac{\nu}{[r(t)]^{2}} a\left(v^{k}, \phi\right)+\frac{\alpha}{r(t)} B\left(v^{k}, v^{k}, \phi\right)-\frac{1}{r(t)} B\left(b^{k}, b^{k}, \phi\right) \\
& =\alpha(J, \phi)+\alpha \frac{r^{\prime}(t)}{r(t)} c\left(v^{k}, \phi\right) \\
& \left(b_{t}^{k}, \psi\right)+\frac{\gamma}{[r(t)]^{2}} a\left(b^{k}, \psi\right)+\frac{1}{r(t)} B\left(v^{k}, b^{k}, \psi\right)-\frac{1}{r(t)} B\left(b^{k}, v^{k}, \psi\right) \\
& =\frac{r^{\prime}(t)}{r(t)} c\left(b^{k}, \psi\right)
\end{aligned}
$$


for all $\phi, \psi \in V^{k}$,

$$
v^{k}(0)=v_{0}^{k} ; b^{k}(0)=b_{0}^{k}
$$

where $v_{0}^{k} \rightarrow v_{0}$ and $b_{0}^{k} \rightarrow b_{0}$ in $H(D)$ as $k \rightarrow \infty$.

Equations (3.7), (3.8) and (3.9) is a system of ordinary differential equations for the coefficient functions $c_{i k}(t)$ and $d_{i k}(t)$, which defines $v^{k}$ and $b^{k}$ in a interval $\left[0, t_{k}[\right.$. We will show some a priori estimates independent of $k$ and $t$, in order to take $t_{k}=T$. Also, we will prove that $\left(v^{k}, b^{k}\right)$ converges in appropriate sense to a solution $(v, b)$ of $(3.3)-(3.6)$. We prove the following lemma.

Lemma 3.1. The transformed system (3.3)-(3.6) admits at least one weak solution $(v, b)$ in $L^{2}(0, T ; V(D)) \cap L^{\infty}(0, T ; H(D))$.

Proof. Setting $\phi=v^{k}$ and $\psi=b^{k}$ in (3.7) and (3.8), respectively, we have

$$
\begin{gathered}
\frac{\alpha}{2} \frac{d}{d t}\left\|v^{k}\right\|^{2}+\frac{\nu}{[r(t)]^{2}}\left\|\nabla v^{k}\right\|^{2}= \\
\alpha\left(J, v_{k}\right)+\frac{1}{r(t)} B\left(b^{k}, b^{k}, v^{k}\right)+\frac{\alpha r^{\prime}(t)}{r(t)} c\left(v^{k}, v^{k}\right) \\
\frac{1}{2} \frac{d}{d t}\left\|b^{k}\right\|^{2}+\frac{\gamma}{[r(t)]^{2}}\left\|\nabla b^{k}\right\|^{2}=\frac{1}{r(t)} B\left(b^{k}, v^{k}, b^{k}\right)+\frac{r^{\prime}(t)}{r(t)} c\left(b^{k}, b^{k}\right)
\end{gathered}
$$

since $B(v, w, w)=0$ for $w \in V^{k}$.

Adding the above inequalities and observing that $\frac{1}{r(t)}\left[B\left(b^{k}, b^{k}, v^{k}\right)+B\left(b^{k}, v^{k}, b^{k}\right)\right]=0$, we obtain

$$
\begin{aligned}
& \frac{1}{2} \frac{d}{d t}\left(\alpha\left\|v^{k}\right\|^{2}+\left\|b^{k}\right\|^{2}\right)+\frac{1}{[r(t)]^{2}}\left(\nu\left\|\nabla v^{k}\right\|^{2}+\gamma\left\|\nabla b^{k}\right\|^{2}\right) \\
& \quad=\alpha\left(J, v^{k}\right)+\frac{r^{\prime}(t)}{r(t)}\left[\alpha c\left(v^{k}, v^{k}\right)+c\left(b^{k}, b^{k}\right)\right] \\
& \quad \equiv F^{k}
\end{aligned}
$$


Now, we use Hölder and Young inequalities to estimate the righthand side of the above inequality, we obtain

$$
\begin{aligned}
\left|F^{k}\right| \leq & \frac{1}{2 \alpha}\|J\|^{2}+\frac{\alpha}{2}\left\|v^{k}\right\|^{2}+C_{\varepsilon}\left|r^{\prime}(t)\right|^{2} \alpha^{2}\|y\|_{L^{\infty}}^{2}\left\|v^{k}\right\|^{2} \\
& +C_{\delta}\left|r^{\prime}(t)\right|^{2}\|y\|_{L^{\infty}}^{2}\left\|b^{k}\right\|^{2}+\frac{\varepsilon}{[r(t)]^{2}}\left\|\nabla v^{k}\right\|^{2} \\
& +\frac{\delta}{[r(t)]^{2}}\left\|\nabla b^{k}\right\|^{2}
\end{aligned}
$$

whence, taking $\varepsilon=\nu / 2$ and $\delta=\gamma / 2$, we arrive at the inequality

$$
\begin{aligned}
& \frac{d}{d t}\left(\alpha\left\|v^{k}\right\|^{2}+\left\|b^{k}\right\|^{2}\right)+\frac{1}{[r(t)]^{2}}\left(\nu\left\|\nabla v^{k}\right\|^{2}+\gamma\left\|\nabla b^{k}\right\|^{2}\right) \\
& \leq \frac{1}{2 \alpha}\|J\|^{2}+\left(C\left|r^{\prime}(t)\right|^{2} \alpha^{2}\|y\|_{L^{\infty}}^{2}+\frac{\alpha}{2}\right)\left\|v^{k}\right\|^{2}+C\left|r^{\prime}(t)\right|^{2}\|y\|_{L^{\infty}}^{2}\left\|b^{k}\right\|^{2} \\
& \leq C\|J\|^{2}+C\left(\alpha\left\|v^{k}\right\|^{2}+\left\|b^{k}\right\|^{2}\right)
\end{aligned}
$$

where $C$ is a positive constant that depends only max $\left\{\left|\gamma^{\prime}(t)\right| \mid 0 \leq t \leq\right.$ $T\}, \alpha,\|y\|_{L^{\infty}}^{2}$.

By integrating the above inequality between 0 and $t$ with $0 \leq t \leq T$, we conclude:

$$
\begin{gathered}
\alpha\left\|v^{k}(t)\right\|^{2}+\left\|b^{k}(t)\right\|^{2}+\int_{0}^{t} \frac{1}{[r(s)]^{2}}\left(\nu\left\|\nabla v^{k}(s)\right\|^{2}+\gamma\left\|\nabla b^{k}(s)\right\|^{2}\right) d s \\
\leq C \int_{0}^{t}\|J(s)\|^{2} d s+C \int_{0}^{t}\left(\alpha\left\|v^{k}(s)\right\|^{2}+\left\|b^{k}(s)\right\|^{2}\right) d s+ \\
\alpha\left\|v^{k}(0)\right\|^{2}+\left\|b^{k}(0)\right\|^{2} .
\end{gathered}
$$


Due to the choice of $v_{0}^{k}$ and $b_{0}^{k}$, there exists $C$ independent of $k$ such that $\left\|v_{0}^{k}\right\| \leq C\left\|v_{0}\right\|,\left\|b_{0}^{k}\right\| \leq C\left\|b_{0}\right\|$, moreover $\int_{0}^{t}\|J(s)\|^{2} d s$ is finite for $0 \leq t \leq T$, we conclude, by using Gronwall's inequality, that

$$
\alpha\left\|v^{k}(t)\right\|^{\dot{2}}+\left\|b^{k}(t)\right\|^{2}+\int_{0}^{t} \frac{1}{[r(s)]^{2}}\left(\nu\left\|\nabla v^{k}(s)\right\|^{2}+\gamma\left\|\nabla b^{k}(s)\right\|^{2}\right) d s \leq C_{1}
$$

Thus, for all $k$ we have that $v^{k}$ and $b^{k}$ exists globally in $t$ and $\left(v^{k}\right)$ and $\left(b^{k}\right)$ remains bounded in $L^{\infty}(0, T ; H(D)) \cap L^{2}(0, T ; V(D))$ as $k \rightarrow \infty$. The next step of the proof consists of proving that $\left(v_{t}^{k}\right)$ and $\left(b_{t}^{k}\right)$ are bounded in $L^{2}\left(0, T ;\left(V_{s}(D)\right)^{\prime}\right)$. To this end, let us fix some notations

$$
\begin{aligned}
& \langle A(t) v, u\rangle=\frac{1}{[r(t)]^{2}} a(v, u) \\
& \langle C(t) v, u\rangle=\frac{r^{\prime}(t)}{r(t)} c(v, u) \\
& \langle E(t) v, u\rangle=\frac{1}{r(t)} B(v, v, u)
\end{aligned}
$$

We will prove that $\left(A(t) v^{k}\right),\left(C(t) v^{k}\right),\left(E(t) v^{k}\right)$ and $\left(E(t) b^{k}\right)$ are bounded in $L^{2}\left(0, T ;\left(V_{s}(D)\right)^{\prime}\right)$.

Indeed, for all $u \in V_{s}$, we have

$$
\begin{aligned}
\left|A\left\langle A(t) v^{k}, u\right\rangle\right| & =\frac{1}{[r(t)]^{2}}\left|a\left(v^{k}, u\right)\right| \\
& \leq \frac{C}{[r(t)]^{2}}\left\|\nabla v^{k}\right\|\|u\|_{s},
\end{aligned}
$$

whence, we have

$$
\left\|A(t) v^{k}\right\|_{\left(V_{s}(D)\right)^{\prime}}=\sup _{\|u\|_{s} \neq 0} \frac{\left|\left\langle A(t) v^{k}, u\right\rangle\right|}{\|u\|_{s}} \leq \frac{C}{[r(t)]^{2}}\left\|\nabla v^{k}\right\|
$$


consequently

$$
\int_{0}^{T}\left\|A(t) v^{k}\right\|_{\left(V_{s}(D)\right)^{\prime}}^{2} d t \leq \int_{0}^{T} \frac{C}{[r(t)]^{2}}\left\|\nabla v^{k}(t)\right\|^{2} d t .
$$

Since $\frac{1}{[r(t)]^{2}} \in C([0, T])$ and $\left(v^{k}\right)$ is bounded in $L^{2}(0, T ; V(D))$, we deduce that

$$
\int_{0}^{T} \frac{C}{[r(t)]^{2}}\left\|\nabla v^{k}(t)\right\|^{2} d t \leq L
$$

and $\left(A(t) v^{k}\right)$ is therefore bounded in $L^{2}\left(0, T ;\left(V_{s}(D)\right)^{\prime}\right)$. Analogously, we show that $\left(C(t) v^{k}\right)$ is bounded in $\left.L^{2}\left(0, T ; V_{s}(D)\right)^{\prime}\right)$. To prove the boundedness of $\left(E(t) v^{k}\right)$ in the space $L^{2}\left(0, T ;\left(V_{s}(D)\right)^{\prime}\right)$ we will use the following interpolation result whose proof can be found in Lions [8]:

Lemma 3.2. If $\left(v^{k}\right)$ is bounded in

$$
L^{2}(0, T ; V(D)) \cap L^{\infty}(0, T ; H(D)),
$$

then $\left(v^{k}\right)$ is also bounded in $L^{4}\left(0, T ; L^{p}(D)\right)$, where $\frac{1}{p}=\frac{1}{2}-\frac{1}{2 n}$.

Using this Lemma, we conclude that

$$
\begin{aligned}
\left|\left\langle E(t) v^{k}, u\right\rangle\right| & \leq \frac{1}{r(t)} \sum_{i, j=1}^{n}\left\|v_{i}^{k}\right\|_{L^{p}}\left\|v_{j}^{k}\right\|_{L^{p}}\left\|\frac{\partial u}{\partial y_{i}}\right\|_{L^{n}} \\
& \leq \frac{1}{r(t)} \sum_{i, j=1}^{n}\left\|v_{i}^{k}\right\|_{L^{p}}\left\|v_{j}\right\|_{L^{p}}\left\|\frac{\partial u}{\partial y_{i}}\right\|_{H^{s-1}} \\
& \leq \frac{C}{\tau(t)}\left\|v^{k}\right\|_{L^{p}}^{2}\|u\|_{s}
\end{aligned}
$$

since $\frac{1}{p}+\frac{1}{p}+\frac{1}{n}=1$ and the Sobolev embedding $H^{s-1}(D) \subseteq L^{n}(D)$. This imply

$$
\int_{0}^{T}\left\|E(t) v^{k}\right\|_{\left(V_{s}(D)\right)^{2}}^{2} d t \leq \int_{0}^{T} \frac{C}{[r(t)]^{2}}\left\|v^{k}(t)\right\|_{L^{p}}^{4} d t .
$$


Since $\frac{1}{[r(t)]^{2}} \in C([0, T])$, we can conclude that $\left(E(t) v^{k}\right)$ is bounded in $L^{2}\left(0, T ;\left(V_{s}(D)\right)^{\prime}\right)$. Similary, we prove that $\left(E(t) b^{k}\right)$ is bounded in $L^{2}\left(0, T ;\left(V_{s}(D)\right)^{\prime}\right)$.

Now, we consider $P_{k}: H \rightarrow V^{k}$ defined by

$$
P_{k} u=\sum_{i=1}^{k}\left(u, \omega^{i}\right) \omega^{i}
$$

since $V_{s}(D) \subset H$ and $V^{k} \subset V_{s}(D)$, we can consider $P_{k}: V_{s}(D) \rightarrow$ $V_{s}(D)$. It is easy to see that $P_{k} \in L\left(V_{s}(D), V_{s}(D)\right),(L(X, Y)$ denote the space of the bounded operator of $X$ into $Y$ ) hence

$$
P_{k}^{*}:\left(V_{s}(D)\right)^{\prime} \rightarrow\left(V_{s}(D)\right)^{\prime}
$$

defined by $\left\langle P_{k}^{*}(v), \omega\right\rangle=\left\langle v, P_{k}(\omega)\right\rangle$ lies in $L\left(\left(V_{s}(D)\right)^{\prime},\left(V_{s}(D)\right)^{\prime}\right)$ and $\left\|P_{k}^{*}\right\| \leq\left\|P_{k}\right\| \leq 1$.

We also observe that the autofunctions $\omega^{i}$ are invariants by $P_{k}$, i.e,

$$
P_{k}\left(\omega^{i}\right)=\omega^{i}
$$

From it and (3.7) we conclude that

$$
\begin{aligned}
\alpha\left(v_{t}^{k}, \omega^{i}\right) & \left.=\left\langle(-\nu A(t)-\alpha E(t)-\alpha C(t)) v^{k}+\alpha J+E(t) b^{k}\right), \omega^{i}\right\rangle \\
& =\left\langle P_{k}^{*}\left((-\nu A(t)-\alpha E(t)-\alpha C(t)) v^{k}+\alpha J+E(t) b^{k}\right), \omega^{i}\right\rangle,
\end{aligned}
$$

whence, for all $\omega \in V_{k}$, we have

$\alpha\left(v_{t}^{k}, \omega\right)=\left\langle P_{k}^{*}\left((-\nu A(t)-\alpha E(t)-\alpha C(t)) v^{k}+\alpha J+E(t) b^{k}\right), \omega\right\rangle$

Hence, by taking $\omega=P_{k} u$, for $u \in V_{s}(D)$, we obtain

$$
\alpha\left(v_{t}^{k}, u\right)=\left\langle P_{k}^{*}\left((-\nu A(t)-\alpha E(t)-\alpha C(t)) v^{k}+\alpha J+E(t) b^{k}\right), u\right\rangle,
$$


and, consequently

$$
\alpha v_{t}^{k}=P_{k}^{*}\left((-\nu A(t)-\alpha E(t)-\alpha C(t)) v^{k}+\alpha J+E(t) b^{k}\right)
$$

belong to $L^{2}\left(0, T ;\left(V_{s}(D)\right)^{\prime}\right)$ thanks to the previous estimates and ||$P_{k}^{*}|| \leq 1$.

Working as before, we have

$$
b_{t}^{k}=P_{k}^{*}\left((-\gamma A(t)-C(t)) b^{k}+H\left(v^{k}, b^{k}\right)-H\left(b^{k}, v^{k}\right)\right),
$$

where $H(u, \omega)=-\frac{1}{r(t)} \tilde{E}(u, \omega)$ and $\langle\tilde{E}(u, \omega), h\rangle=B(u, \omega, h)$. Consequently, it is sufficient to show that $H\left(v^{k}, b^{k}\right)$ and $H\left(b^{k}, v^{k}\right)$ belong to $L^{2}\left(0, T ;\left(V_{s}(D)\right)^{\prime}\right)$ to conclude that $b_{t}^{k}$ is bounded in $L^{2}\left(0, T ;\left(V_{s}(D)\right)^{\prime}\right)$. We observe that

$$
\int_{0}^{T}\left\|H\left(v^{k}, b^{k}\right)\right\|_{\left(V_{s}(D)\right)^{\prime}}^{2} d t=\int_{0}^{T} \frac{1}{r(t)}\left\|\tilde{E}\left(v^{k}, b^{k}\right)\right\|_{\left(V_{s}(D)\right)^{\prime}}^{2} d t
$$

On the other hand,

$$
\begin{aligned}
\left|\left\langle\tilde{E}\left(v^{k}, b^{k}\right), h\right\rangle\right| & \leq \sum_{i, j}\left\|v_{j}^{k}\right\|_{L_{\mathbf{p}}}\left\|b_{i}^{k}\right\|_{L^{p}}\left\|\frac{\partial h}{\partial y_{i}}\right\|_{L^{n}} \\
& \leq C\left\|v^{k}\right\|_{L^{\mathbf{p}}}\left\|b^{k}\right\|_{L^{\mathbf{p}}}\|h\|_{s}
\end{aligned}
$$

and therefore

$$
\left\|\tilde{E}\left(v^{k}, b^{k}\right)\right\|_{\left(V_{s}(D)\right)^{\prime}}^{2} \leq C\left\|v^{k}\right\|_{L^{p}}^{2}\left\|b^{k}\right\|_{L^{p}}^{2}
$$


this imply

$$
\begin{aligned}
& \int_{0}^{T} \frac{1}{r(t)}\left\|\tilde{E}\left(v^{k}, b^{k}\right)\right\|_{\left(V_{d}(D)\right)^{\prime}}^{2} \leq \\
& C\left(\int_{0}^{T} \frac{1}{[r(t)]^{2}}\left\|v^{k}\right\|_{L^{p}}^{4}\right)^{1 / 2}\left(\int_{0}^{T} \frac{1}{[r(t)]^{2}}\left\|b^{k}\right\|_{L^{p}}^{4}\right)^{1 / 2} \cdot \leq C
\end{aligned}
$$

Similarly, we prove that $H\left(b^{k}, v^{k}\right)$ is bounded in $L^{2}\left(0, T ;\left(V_{s}(D)\right)^{\prime}\right)$.

Therefore, arguing as in the book of Lions $[8$, p. 76] and making use of the Aubin-Lions Lemma with $B_{0}=V(D), p_{0}=2, B_{1}=$ $\left(V_{s}(D)\right)^{\prime}, p_{1}=2$ and $B=H(D)$ (see Theorem 1.5.1 and Lemma 1.5 .2 of the above book, p. 58), we can conclude that there exist $v, b \in$ $L^{2}(0, T ; V(D))$ such that, up to a subsequence which we shall denote again by the suffix $k$, there hold

$$
\begin{aligned}
& \left.\begin{array}{l}
v^{k} \rightarrow v \\
b^{k} \rightarrow b
\end{array}\right\} \quad \text { in } L^{2}(0, T ; V(D)) \text { and } L^{\infty}(0, T ; H(D)) \text { weakly and } \\
& \left.\begin{array}{l}
v^{k} \rightarrow v \\
b^{k} \rightarrow b
\end{array}\right\} \quad \text { in } L^{2}(0, T ; H(D)) \text { strongly, as } k \rightarrow . \infty \\
& \left.\begin{array}{l}
v_{t}^{k} \rightarrow v_{t} \\
b_{t}^{k} \rightarrow b_{t}
\end{array}\right\} \quad \text { in } L^{2}\left(0, T ;\left(V_{s}(D)\right)^{\prime} \text { weakly, as } k \rightarrow \infty\right.
\end{aligned}
$$

Now, the next step is to take the limit. But, once the above convergence results have been established, this is standard procedure and it follows the same patter as in Lions [8, p. 76-77]. Consequently, we 
244

M.A. Rojas-Medar and R. Beltrán-Barrios

shall omit it and we will directly deduce that

$$
\begin{aligned}
& -\int_{0}^{T} \alpha\left(v, \phi^{\prime}\right)+\int_{0}^{T} \frac{\nu}{[r(t)]^{2}} a(v, \phi)+\int_{0}^{T} \frac{\alpha}{r(t)} B(v, v, \phi)- \\
& \int_{0}^{T} \frac{1}{r(t)} B(b, b, \phi)=\int_{0}^{T} \alpha(J, \phi)+\int_{0}^{T} \alpha \frac{r^{\prime}(t)}{r(t)} c(v, \phi)
\end{aligned}
$$

and

$$
\begin{gathered}
-\int_{0}^{T} \alpha\left(v, \psi^{\prime}\right)+\int_{0}^{T} \frac{\gamma}{[r(t)]^{2}} a(b, \psi)+\int_{0}^{T} \frac{1}{r(t)} B(v, b, \psi)- \\
\int_{0}^{T} \frac{1}{r(t)} B(b, v, \psi)=\int_{0}^{T} \frac{r^{\prime}(t)}{r(t)} c(b, \phi)
\end{gathered}
$$

for all $\phi, \psi \in C^{1}(U)$ such that $\operatorname{div} \phi=\operatorname{div} \psi=0$. So, the Lemma is proved.

To conclude the proof of Theorem, let us consider a test function $\varphi \in C_{0}^{1}(U)$ such that div $\varphi=0$, and define

$$
\phi(y, t)=[r(t)]^{n} \varphi(y r(t), t) .
$$

Integrating by parts,

$$
-\int_{0}^{T} \alpha\left(v, \phi^{\prime}\right)-\int_{0}^{T} \alpha \frac{r^{\prime}(t)}{r(t)} c(v, \phi)=-\int_{0}^{T} \alpha[r(t)]^{n}\left(v, \varphi^{\prime}\right)
$$


and also

$$
\begin{aligned}
& \int_{0}^{T} \frac{\nu}{[r(t)]^{2}} a(v, \phi)=\sum_{i=1}^{n} \int_{0}^{T} \int_{D} \nu \frac{[r(t)]^{n}}{r(t)} \frac{\partial v}{\partial y_{i}} \frac{\partial \varphi}{\partial y_{i}} \\
& \int_{0}^{T} \frac{\alpha}{r(t)} B(v, v, \phi)=-\sum_{i, j=1}^{n} \int_{0}^{T} \int_{D} \alpha[r(t)]^{n} v_{i} \frac{\partial \varphi_{i}}{\partial y_{i}} v_{j} \\
& \int_{0}^{T} \frac{1}{r(t)} B(b, b, \phi)=-\sum_{i, j=1}^{n} \int_{0}^{T} \int_{D}[r(t)]^{n} b_{i} \frac{\partial \varphi_{i}}{\partial y_{i}} b_{j} .
\end{aligned}
$$

By using the above identities in (3.11), we obtain,

$$
\begin{aligned}
& -\int_{0}^{T} \int_{D} \alpha[r(t)]^{2} v(y, t) \varphi^{\prime}(y r(t), t)+\sum_{i=1}^{n} \int_{0}^{T} \int_{D} \nu \frac{\left[r(\underline{t}]^{n}\right.}{r(t)} \frac{\partial v}{\partial y_{i}} \frac{\partial \varphi}{\partial y_{i}} \\
& -\sum_{i=1}^{n} \int_{0}^{T} \int_{D} \alpha[r(t)]^{n} v_{i} v_{j} \frac{\partial \varphi_{j}}{\partial y_{i}}+\sum_{i=1}^{n} \int_{0}^{T} \int_{D}[r(t)]^{n} b_{i} b_{j} \frac{\partial \varphi_{i}}{\partial y_{i}} \\
& =\int_{0}^{T} \int_{D} \alpha[r(t)]^{n} J \varphi .
\end{aligned}
$$

Analagously, we obtain for $b$,

$$
\begin{aligned}
& -\int_{0}^{T} \int_{D}[r(t)]^{n} b(y, t) \tilde{\varphi}^{\prime}(y r(t), t)+\sum_{i=1}^{n} \int_{0}^{T} \int_{D} \frac{[r(t)]^{n}}{r(t)} \frac{\partial b}{\partial y_{i}} \frac{\partial \tilde{\varphi}}{\partial y_{i}} \\
& -\int_{0}^{T} \int_{D}[r(t)]^{n} v_{i} b_{j} \frac{\partial \tilde{\varphi}_{j}}{\partial y_{i}}+\int_{0}^{T} \int_{D}[r(t)]^{n} b_{i} v_{j} \frac{\partial \tilde{\varphi}_{j}}{\partial y_{i}}=0
\end{aligned}
$$

where $\tilde{\varphi} \in C_{0}^{1}(U)$ with $\operatorname{div} \tilde{\varphi}=0$. 
Let us now consider the transformation $\tau^{-1}: U \rightarrow Q$ which is defined by (3.1). We observe that its Jacobian is $[r(t)]^{n}$. Consequently, by change of variables in the integrals, (3.13) and (3.14) become

$$
\begin{aligned}
-\int_{Q} \alpha u \varphi^{\prime}+\sum_{i=1}^{n} \int_{Q} \nu \nabla u_{i} \nabla \varphi_{i} & -\sum_{i, j=1}^{n} \int_{Q} u_{j} \frac{\partial \varphi_{i}}{\partial x_{j}} u_{i} \\
& +\sum_{i, j=1}^{n} \int_{Q} h_{j} \frac{\partial \varphi_{i}}{\partial x_{j}} h_{i}=\int_{Q} \alpha f \cdot \varphi
\end{aligned}
$$

and

$-\int_{a} h \cdot \tilde{\varphi}^{\prime}+\sum_{i=1}^{n} \int_{Q} \gamma \nabla h_{i} \nabla \tilde{\varphi}_{i}-\sum_{i, j=1}^{n} \int_{Q} u_{j} \frac{\partial \tilde{\varphi}_{i}}{\partial x_{j}} h_{i}+\sum_{i, j=1}^{n} \int_{Q} h_{j} \frac{\partial \tilde{\varphi}_{i}}{\partial x_{j}} u_{i}=0$

which proves that $(u, h)$ is a weak solution of the problem; since the mappings

$$
\begin{aligned}
L^{2}(0, T ; V(D)) & \rightarrow L^{2}(0, T ; V(Q(t))) \\
v(y, t) & \rightarrow u(x, t)=v\left(\frac{x}{r(t)}, t\right), \\
b(y, t) & \rightarrow h(x, t)=h\left(\frac{x}{r(t)}, t\right),
\end{aligned}
$$

and

$$
\begin{aligned}
L^{2}(0, T ; H(D)) & \rightarrow L^{2}(0, T ; H(Q(t)) \\
v(y, t) & \rightarrow u(x, t)=v\left(\frac{x}{r(t)}, t\right) \\
b(y, t) & \rightarrow h(x, t)=b\left(\frac{x}{r(t)}, t\right)
\end{aligned}
$$


are smooth bijections of class $C^{1}$, it follows that

$$
u, h \in L^{2}(0, T ; V(Q(t))) \cap L^{\infty}(0, T ; H(Q(t))) .
$$

Finally, a standard arguments shows that $u(0)=u_{0}$ and $h(0)=h_{0}$ (see remark 2.2). This finished the proof of the Theorem.

\section{PROOF OF THEOREM 2.4 .}

We first prove the regularity result. We observe that the proof of the above theorem shown that $u^{\prime} \in L^{2}\left(0, T ; V^{\prime}\right)$; consequently, applying Lemma 1.2 in Temam [15], p. 260, we obtain that $u$ is almost everywhere equal to a function continuous from $[0, T]$ into $H$.

Thus,

$$
u \in C([0, T] ; H)
$$

and (2.2) follows easily. Analogously it is proved the continuity of $h$ and (2.3).

We also recall that Lemma 1.2 in Temam [15], p. 260-261, asserts that the equations below holds:

$$
\begin{aligned}
& \frac{d}{d t}\|u(t)\|^{2}=2\left\langle u^{\prime}(t), u(t)\right\rangle, \\
& \frac{d}{d t}\|h(t)\|^{2}=2\left\langle h^{\prime}(t), h(t)\right\rangle .
\end{aligned}
$$

These results will be used in the following proof of uniqueness which we will start now.

Consider that $\left(u_{1}, h_{1}\right)$ and $\left(u_{2}, h_{2}\right)$ are two solutions of the problem (1.1)-(1.3) with the same $f$ and $u_{0}, h_{0}$ and define the differences $\omega=$ 
$u_{1}-u_{2}$ and $v=h_{1}-h_{2}$. They satisfy

$$
\begin{aligned}
\alpha\left(\omega_{t}, \phi\right)+\nu a(\omega, \phi) & =-\alpha B\left(\omega, u_{1}, \phi\right)-\alpha B\left(u_{2}, \omega, \phi\right) \\
& +B\left(v, h_{1}, \phi\right)+B\left(h_{2}, v, \phi\right) \\
\left(v_{t}, \psi\right)+\gamma a(v, \psi) & =-B\left(u_{1}, v, \psi\right)-B\left(\omega, h_{2}, \psi\right) \\
& +B\left(v, u_{1}, \psi\right)+B\left(h_{2}, \omega, \psi\right)
\end{aligned}
$$

for any $\phi, \psi \in V$; also $\omega(0)=v(0)=0$.

By the proof of Theorem 2.3, $w_{t}$ and $v_{t}$ belong to $L^{2}\left(0, T ; V^{\prime}\right)$; consequently by setting $\phi=w$ and $\psi=v$ in the above inequalities, we obtain

$$
\begin{gathered}
\frac{\alpha}{2} \frac{d}{d t}\|\omega\|^{2}+\nu a(\omega, \omega)=-\alpha B\left(\omega, u_{1}, \omega\right)+B\left(v, h_{1}, \omega\right)+B\left(h_{2}, v, \omega\right) \\
\frac{1}{2} \frac{d}{d t}\|v\|^{2}+\gamma a(v, v)=-B\left(\omega, h_{2}, v\right)+B\left(v, u_{1}, v\right)+B\left(h_{2}, \omega, v\right)
\end{gathered}
$$

thanks to the above remark.

Adding the above identities, we get

$$
\begin{aligned}
\frac{1}{2} & \frac{d}{d t}\left(\alpha\|\omega\|^{2}+\|v\|^{2}\right)+\nu\|\omega\|_{1}+\gamma\|v\|_{1} \\
\quad & =-\alpha B\left(\omega, u_{1}, \omega\right)+B\left(v, h_{1}, \omega\right)-B\left(\omega, h_{2}, v\right)+B\left(v, u_{1}, v\right)
\end{aligned}
$$

since $B\left(h_{2}, v, \omega\right)+B\left(h_{2}, \omega, v\right)=0$. 
Now, we observe that

$$
\begin{aligned}
\alpha B\left(\omega, u_{1}, \omega\right) & \leq \alpha C\|\omega\|_{L^{4}}^{2}\left\|u_{1}\right\|_{1} \\
& \leq \alpha^{2} C\|\omega\|\|\omega\|_{1}\left\|u_{1}\right\|_{1} \\
& \leq \frac{\nu}{6}\|\omega\|_{1}^{2}+C_{\nu}(\alpha) \alpha\|\omega\|^{2}\left\|u_{1}\right\|_{1}^{2}
\end{aligned}
$$

where we used the Lemma 3.3 in Temam [15], p. 291, together with Hölder and Young inequalities.

Analogously, we can prove

$$
\begin{aligned}
& B\left(v, u_{1}, v\right) \leq \frac{\gamma}{6}\|v\|_{1}^{2}+C_{\gamma}\|v\|^{2}\left\|u_{1}\right\|_{1}^{2}, \\
& B\left(v, h_{1}, \omega\right) \leq \frac{\nu}{6}\|\omega\|_{1}^{2}+\frac{\gamma}{6}\|v\|_{1}^{2}+C_{\nu, \gamma}(\alpha)\left(\|v\|^{2}+\alpha\|\omega\|^{2}\right)\left\|h_{1}\right\|_{1}^{2} \\
& B\left(\omega, h_{2}, v\right) \leq \frac{\nu}{6}\|\omega\|_{1}^{2}+\frac{\gamma}{6}\|v\|^{2}+C_{\nu, \gamma}(\alpha)\left(\|v\|^{2}+\alpha\|\omega\|^{2}\right)\left\|h_{2}\right\|_{1}^{2} .
\end{aligned}
$$

By using the above inequalities in (4.1), we get

$$
\begin{aligned}
& \frac{d}{d t}\left(\alpha\|\omega\|^{2}+\|v\|^{2}\right)+\nu\|\omega\|_{1}^{2}+\gamma\|v\|_{1}^{2} \\
& \leq C\left(\alpha\|\omega\|^{2}+\|v\|^{2}\right)\left(\left\|u_{1}\right\|_{1}^{2}+\left\|h_{1}\right\|_{1}^{2}+\left\|h_{2}\right\|_{1}^{2}\right),
\end{aligned}
$$

where $C$ is a positive constant that only depend on $\nu, \gamma, \alpha$.

By integrating in time, the use of Gronwall's inequality, we obtain

$$
\alpha\|\omega(t)\|^{2}+\|v(t)\|^{2} \leq\left(\alpha\|\omega(0)\|^{2}+\|v(0)\|^{2}\right) e^{\varphi(t)}
$$


where $\varphi(t)=C \int_{0}^{t}\left(\left\|u_{1}\right\|_{1}^{2}+\left\|h_{1}\right\|_{1}^{2}+\left\|h_{2}\right\|_{1}^{2}\right) d s<+\infty$, for every $t \in[0, T]$. This last inequality, implies $\omega(t)=v(t)=0$. Hence $u_{1}=u_{2}$ and $h_{1}=h_{2}$. and the uniqueness is proved. This completes the proof of the Theorem.

\section{References}

[1] Adams, R.A.: Sobolev Spaces, Academic Press, New York, 1975.

[2] Boldrini, J.L. and Rojas-Medar, M.A.: On a system of evolution equations of magnetohydrodynamic type. To appear in Matemática Contemporánea.

[3] Conca, C. and Rojas-Medar, M.A.: The initial value problem for the Boussinesq equations in a time-dependent domain, Informe Interno, M.A.-993-B-402, Universidad de Chile, Chile, 1993, submitted.

[4] Dal Passo, R. et Ughi, M.: Problème de Dirichlet pour une classe d'équations paraboliques non linéaires dans des ouverts non cylindriques, C.R. Acad. Sc. Paris Série I, 308 (1989), 555-558.

[5] Fujita, H. and Kato, T.: On the Navier-Stokes initial value problem, I, Arch Rational Mech. Anal., 16 (1964), 269-315.

[6] Fujita, H. and Sauer, N.: Construction of weak solutions of the Navier-Stokes equations in a non-cylindrical domain, bull. Amer. Math. Soc., 75 (1969), 465-468.

[7] Lassner, G.: Über ein rand-anfangswert-problem der magnetohydrodinamik, Arch. Rational Mech. Anal., 25 (1967), 388-405.

[8] Lions, J.L.: Une remarque sur les problèmes d'evolution non linéaires dans des domains non cylindriques, Rev. Roumaine Math. Pures Appl., 9 (1964), 11-18.

[9] Lions, J.L.: Quelques méthodes de résolution des problèmes aux limites non-lineaires, Dunod Gauthier-Villars, Paris, 1969.

[10] Ōeda, K.: On the initial problem for the heat convection equation of Boussinesq approximation in a time-dependent domain, Proc. Japan Acad. Ser. A. Math Sci., 25 (1988), 143-146.

[11] Ôtani, M. and Yamada, Y.: On the Navier-Stokes equations in noncylindrical domains: An approach by the subdifferential operator theory, Fac. Sci. Univ. Tokyo Sect IA, 25 (1978), 185-204. 
[12] Pikelner, S.B.: Grundlander der Kosmischen elktrodynamic, Moscou, 1966 .

[13] Rojas-Medar, M.A. and Boldrini, J.L.: Global strong solutions of equations of magnetohydrodynamic type, Relatório de Pesquisa, R.P. 53, IMECC-UNICAMP, Brazil, 1993, submitted.

[14] Schlüter, A.: Dynamic des plasmas, I and II, Z. Naturforsch. 5a, (1950), 72-78; 6a., (1951), 73-79.

[15] Temam, R.: Navier-Stokes equations, North-Holland, Amsterdam, Rev. Edit., 1979.

UNICAMP IMECC, CP6065

13081-970, Campinas, SP

Brazil

\author{
Departamento de Matemática, \\ Universidad de Tarapacà \\ Casilla 7D, Arica \\ Chile
}

Recibido: 27 de Octubre de 1993 